\title{
Different Patterns of Attention Bias in Worry and Rumination
}

\author{
Juyoen Hur ${ }^{1}$, Kelly Gaul $^{2}$, \& Howard Berenbaum ${ }^{3}$ \\ ${ }^{1}$ Department of Psychology, University of Maryland, College Park, MD, 20742, USA \\ ${ }^{2}$ Department of Psychology, Roosevelt University, Chicago, IL, 60605, USA \\ ${ }^{3}$ Department of Psychology, University of Illinois at Urbana-Champaign, Champaign, IL, 61820, \\ USA
}

Running head: Attentional Biases, Worry and Rumination

\section{Corresponding Author:}

Juyoen Hur

Department of Psychology

University of Maryland, College Park

4094 Campus Dr.

College Park, MD, 20742, USA

Ph: (617) 955-5452

Email: juh614@gmail.com

Keywords: Worry, Rumination, Attentional Bias, Threat, Loss

Author Note: This study is based in part on a dissertation submitted by Juyoen Hur to the Graduate College of the University of Illinois at Urbana-Champaign. 


\begin{abstract}
In two studies with college student participants, we explored the ways in which worry and rumination may be similar or distinct. Towards that end, as part of our research, we developed new laboratory measures of worry and rumination. In Study 1, we examined how the new lab instruments we developed differentiate worry vs. rumination and initiation vs. termination. We did so by comparing them with two other measurement methods of worry and rumination, ecological momentary assessment and questionnaires. We found that the laboratory measures were reasonably able to differentiate worry from rumination, but not initiation from termination. In Study 2, we further examined the relationship between attentional biases to threat/danger and loss/failure (using the dot probe task) and both worry and rumination to explore the nature of distinctions between worry and rumination. We found different patterns of attentional bias associated with worry and rumination. Worry was associated with bias away from threat. In contrast, rumination was associated with bias toward loss/failure. The results of the two studies suggest that there are some meaningful differences between worry and rumination. The implications of the current findings are discussed.
\end{abstract}


Attentional Biases, Worry and Rumination

\section{INTRODUCTION}

Worry and rumination are examples of maladaptive repetitive thinking that are important vulnerability factors for, and key features of, emotional disorders such as generalized anxiety disorder (GAD) and major depressive disorder (MDD) (Ruscio, Seitchik, Gentes, Jones, \& Hallion, 2011; Smith \& Alloy, 2009; Watkins, 2008). There have been fruitful discussions and debates about the mechanisms and nature of worry and rumination. However, more work needs to be done, especially regarding the nature of distinctions and similarities between worry and rumination (Beck, Brown, Steer, Eidelson, \& Riskind, 1987; Clark, Beck, \& Brown, 1989; Segerstrom, Stanton, Alden, \& Shortridge, 2003; Watkins, 2008), as well as understanding their temporal dynamics (Berenbaum, 2010; Oathes, Siegle, \& Ray, 2011). The goal of the present research was to further explore the ways in which worry and rumination are distinct and similar; toward that end, as part of our research, we developed new laboratory measures of worry and rumination.

In recent years, a good bit of work has drawn attention to common characteristics shared by worry and rumination (McEvoy, Watson, Watkins, \& Nathan, 2013; Ruscio et al., 2011; Segerstrom et al., 2003; Watkins, 2008). For example, both worry and rumination are primarily verbal-linguistic and involve self-focused negative thoughts (Mor \& Winquist, 2002; Stokes \& Hirsch, 2010). With an increased focus on the transdiagnostic mechanisms underlying cognitiveaffective dysfunctions among mood and anxiety disorders, recent research proposes that worry and rumination represent a common core process of perseverative thought and a tendency to engage in negative thinking in a repetitive, uncontrolled manner (Segerstrom et al., 2003; Watkins, 2008). Similarly, recent studies found that "negative repetitive thinking" is a common 
risk factor for the emotional difficulties observed in both GAD and MDD (McEvoy et al., 2013; Ruscio et al., 2011; Watkins, 2008).

Although work in recent years clearly demonstrates important similarities between worry and rumination, there still may be some important differences. Historically, researchers considered worry and rumination as two distinct entities (Beck et al., 1987; Clark et al., 1989), proposing that worry is mostly concerned with future events that entail possible threatening outcomes (Berenbaum, 2010), whereas rumination is mostly concerned with past events, especially regarding past loss or failure (Kirkegaard Thomsen, 2006; Nolen-Hoeksema, Wisco, \& Lyubomirsky, 2008; Segerstrom et al., 2003). The avoidance theory of worry (Borkovec, 1994) posits that worry is a cognitive avoidance response to perceived threat, whereas goal-fulfillment and discrepancy models of rumination (Martin, Tesser, \& McIntosh, 1993; Wanke \& Schmid, 1996) posit that a failure or loss in making progress towards personal goals drives ruminative thinking. The present research is grounded in the expectation that there are both similarities and differences between worry and rumination, and what needs to be done is to explicate what they are.

One way in which worry and rumination may differ is the attentional mechanisms involved. Cognitive models posit that emotional disorders are caused and maintained by biases in the processing of emotion-congruent information (Beck \& Haigh, 2014), and numerous studies have found links between attentional biases and anxiety and depression (Koster, De Raedt, Goeleven, Franck, \& Crombez, 2005; Mathews \& MacLeod, 2005; Mogg, Bradley, Williams, \& Mathews, 1993). In addition, although it still is a matter of debate, the results of previous research suggest that worry is correlated more strongly with anxiety than with depression, whereas rumination is correlated more strongly with depression than anxiety (Beck et al., 1987; 
Kendall \& Ingram, 1989). Thus, delineating the attentional mechanisms involved in worry and rumination can provide new insights into the underlying mechanisms of the development of anxiety and depression and set the stage for developing improved interventions.

Despite its theoretical and clinical implications, studies have only recently begun to examine the direct relationship between worry and rumination and attentional biases. The few studies that directly investigated the relationship between worry and attention bias have yielded mixed results. Whereas some found evidence of attentional bias towards threat in high trait anxious groups or high worriers (Bar-Haim, Lamy, Pergamin, Bakermans-Kranenburg, \& Van Ijzendoorn, 2007; Goodwin, Eagleson, Mathews, Yiend, \& Hirsch, 2017), others failed to find an association between attentional bias to threat and worry (Engels et al., 2007; Oathes et al., 2011; Sass et al., 2010). Similarly, an association between rumination and attention bias is largely unsettled. Some studies found a significant association between the two when the dysphoric stimuli were presented for a long duration (e.g., 1000ms) (Donaldson, Lam, \& Mathews, 2007; Joormann, Dkane, \& Gotlib, 2006), whereas other studies suggest that rumination is specifically related to impaired attentional disengagement from, rather than facilitated engagement toward, negative information (Grafton, Southworth, Watkins, \& MacLeod, 2016; Southworth, Grafton, MacLeod, \& Watkins, 2017). In summary, though inconclusive, past research raises the possibility that worry and rumination have different patterns of attentional bias, with the two potentially differing with respect to: (a) the content of stimuli to which attention is biased (i.e., content-specificity); and (b) whether attention is biased toward or away from specific types of stimuli.

Prior research on worry and rumination has focused almost exclusively on the content and amount of worrying/ruminating. For example, questionnaires, the most common method 
used to measure worry and rumination, assess worry and rumination using items such as "I worry all the time" and "I spend a great deal of time thinking back over my embarrassing or disappointing moments.” More recently, however, Berenbaum (2010) proposed an initiationtermination (IT) two-phase model of worrying in which the initiation and termination phases of worry are differentiated. He argued that worry should not be considered a static entity, but should instead be thought of as a dynamic process that unfolds over time (Berenbaum, 2010). The IT model suggests that the following aspects of worrying need more attention: (a) how easily worrying is initiated; and (b) how easily worrying is terminated. According to the IT model, worry is initiated by perceptions of threat, whereas worrying is terminated when the threat is accepted. To the extent that an individual is unable or unwilling to accept the threat, s/he is unlikely to stop worrying. An initial test of the IT model of worry (Berenbaum et al., 2018) using ecological momentary assessment (EMA) found that ease of worry initiation and difficulty with worry termination incrementally predicted global worry and GAD symptom severity, highlighting the potential value of distinguishing between the initiation and termination of worrying. Although Berenbaum's (2010) model was formulated in relation to worry, it is possible to extend the model to rumination.

Although questionnaires and interviews are most commonly used for assessing worry and rumination (Meyer, Miller, Metzger, \& Borkovec, 1990; Sheehan et al., 2001; Trapnell \& Campbell, 1999), they are limited in that they are retrospective reports that can be influenced by a variety of recall biases (Hufford, 2007). In addition, none of them distinguishes the initiation vs. termination phases of worry and rumination. An alternative strategy is laboratory tasks. Laboratory tasks can minimize many sources of recall bias, and provide the opportunity to examine the pattern of individuals' emotional, behavioral, and cognitive experiences in a 
controlled environment. Several studies have used laboratory tasks to measure individuals' worry and rumination patterns. For example, repetitive negative thoughts were measured via thought sampling during a focused breathing task (FBT; Borkovec, Robinson, Pruzinsky, \& DePree, 1983; Ruscio \& Borkovec, 2004). During 5 minutes of FBT, participants were signaled four times at varying intervals (from 30 to 120 seconds apart); at each signal, they answered a set of questions that assessed whether they were engaging in negative thoughts at the moment.

Although FBT showed good predictive validity (Ruscio et al., 2011), it remains unclear whether what is captured during FBT is specific to the ease of initiating negative thoughts, difficulty terminating those thoughts, or both. In addition, studies using FBT did not differentiate worry and rumination, but rather assessed negative automatic thoughts. In other studies (Eysenck, 1984; Rapee, 1993), worry was measured during a thinking period during which participants were instructed to simply worry about the worry topic they had previously identified. During the 5minute thinking period, an auditory stimulus (e.g., beep sound) was presented every 15 seconds and participants answered whether they had been worrying when the signal occurred. Although Eysenck (1984) showed the modulation effect by trait and state negative affect on different time points of the worry process (e.g., initial 75 seconds vs. later), his experiment was not designed to capture individual differences in worry initiation and termination; further, rumination was not assessed. In Study 1, we modified the tasks that had been developed by Borkovec et al. (1983) and Eysenck (1984) to create new laboratory instruments to explore distinctions and similarities between worry and rumination as well as those between initiation vs. termination.

The goals of the present research were to: (1) explore the ways in which worry and rumination may be similar or distinct, particularly focusing on the pattern of their associations with attentional biases; and 2) develop and examine lab instruments to help us achieve the first 
goal. In Study 1, we examined how the new lab instruments we developed differentiate worry vs. rumination and initiation vs. termination. We did so by comparing the new lab instruments with two other measurement methods of worry and rumination, EMA and questionnaires. In Study 2, we examined the relationship between attentional biases to threat/danger and loss/failure (using the dot probe task) and both worry and rumination to explore potential distinctions between worry and rumination. Existing conceptualizations describe worry as repeated ideation focused on to-be-avoided threats, and describe rumination as focused on to-be-understood loss or failure (Mathews \& MacLeod, 2005; Smith \& Alloy, 2009). As such, we hypothesized that worry would be associated with biased attention to threat, rather than loss/failure, whereas rumination would be associated with biased attention to loss/failure rather than threat. Per direction of attention biases (orienting vs. avoidance), we had no a priori hypothesis. Rather, the literature suggests two competing predictions. On the one hand, based on prior findings that high worriers and individuals with GAD show an increased attention bias towards threat (Goodwin, Yiend, \& Hirsch, 2017; Mathews \& MacLeod, 2005), one might expect worry to be associated with biased attention towards threat. On the other hand, based on the avoidance theory of worry (Borkovec, 1994), which posits that worry is a cognitive avoidance response to perceived threat, one might expect worry to be associated with biased attention away from threat. Per rumination, based on some of the prior findings that high ruminators and individuals with MDD show an increased attentional bias towards dysphoric information (Donaldson et al., 2007; Joormann et al., 2006), one might expect that rumination to be associated with biased attention towards loss/failure. On the other hand, based on the possibility that, like worry, rumination may be a cognitive avoidance strategy to perceived loss/failure, one might expect rumination to be associated with biased attention away from threat. 


\section{STUDY 1}

In Study 1, we designed separate lab tasks to measure each of the following: worry initiation, worry termination, rumination initiation, and rumination termination. The same constructs were measured using EMA, which involves repeated sampling of participants' behaviors and experiences in real time in the contexts in which they naturally occur (Shiffman, Stone, \& Hufford, 2008). Past research has shown that EMA reasonably distinguishes between the initiation of worry and the termination of worry (Berenbaum et al., 2018). In addition, we administered well-established worry and rumination questionnaires (i.e., PSWQ, RRQ). In Study 1, we aimed to examine how the new lab instruments we developed differentiate worry vs. rumination and initiation vs. termination by comparing them with questionnaires and EMA.

\section{METHOD}

\section{Participants}

Participants were 46 undergraduate students (66.7\% female; mean age $=20.3$ years). The majority of participants (59.1\%) were White, followed by $29.5 \%$ Asian, and $9.1 \%$ African American; $2.3 \%$ chose to describe themselves as "other." All participants completed a voluntary informed consent at the start of the session and received monetary compensation for their participation. The research protocol was approved by the university institutional review board. Participants were tested individually.

\section{Procedures}

We used a within-subject design in which participants completed all of the following tasks: four laboratory tasks measuring the initiation and termination of worry and rumination, EMA, and questionnaires. To minimize fatigue and potential transfer effects between lab tasks, the study was conducted in two separate sessions, in such a way that rumination and worry lab 
tasks were administered on a different day. The order of tasks were counterbalanced, such that half of the participants completed rumination lab tasks on the first day while completing the worry lab tasks on the second day, and the other half started with worry tasks. The order of administering worry/rumination questionnaires was also randomized. Participants completed the EMA after they completed the lab sessions.

\section{Self-Report Questionnaires}

Worry was measured using the Penn State Worry Questionnaire (PSWQ; Meyer et al., 1990). Participants rated how each of 16 statements described them (e.g., "My worries overwhelm me") on a scale from 1 (not at all typical) to 5 (very typical). There was no missing data and the internal consistency in our sample was excellent $(\alpha=.90)$.

Rumination was measured using the rumination subscale of Rumination/Reflection Questionnaire (RRQ; Trapnell \& Campbell, 1999). Participants rated how each of 12 statements described them (e.g., I tend to ruminate or dwell over things that happened to me for a really long time afterward") on a scale from 1 (strongly disagree) to 5 (strongly agree). There was no missing data and the internal consistency in our sample was good $(\alpha=.87)$.

\section{Laboratory Assessment of Initiation and Termination of Worry/Rumination}

The initiation lab tasks included a shortened version of the FBT used by Ruscio and Borovec (2004) but with a brief priming phase preceding the FBT. The priming phase was added: (1) to capture a wide range of initiation proneness; and (2) to prime for worry vs. rumination specifically. In addition, to enable us to specifically measure worry initiation, we developed a new way of scoring the task (detailed in Methods). The termination lab tasks were developed by modifying Eysenck’s (1984) worry task. Similar to Eysenck’s worry task, we instructed participants to simply worry about the worry topic they had previously identified. However, at 
each prompt during the task, instead of asking participants whether they had been worrying when the signal occurred, we asked whether they had been worrying continuously since the last signal, and how difficult they found it to stop worrying. Detailed information about each task is described below.

\section{Worry Initiation Task}

To measure worry initiation, participants engaged in three trials of a quiet time (QT) task, each of which was preceded by a brief priming phase. The QT task was adapted from the Focused Breathing Task by Ruscio and Borkovec (2004). During the QT, which lasted one minute, participants were instructed to close their eyes and pay attention to their breathing. They were signaled twice (first after 30 seconds, and then again after 1 minute) and, at each signal, participants were first asked to indicate whether they had been engaging in some thoughts at least some of the time during the preceding 30 seconds. If they answered affirmatively, they were asked the following questions: (a) whether these thoughts were positive, negative, or neutral; (b) whether what they were thinking about concerned something that happened in the past; and (c) whether what they were thinking about something that could happen in the future. Participants who indicated that they were thinking about something negative that could happen in the future were judged to have initiated worry. After answering questions following the first signal, participants were once again asked to close their eyes and pay attention to their breathing.

A brief priming phase preceded the QT task in order to avoid obtaining skewed data (i.e., a lot of individuals showing no initiation of worry) and to capture a wide range of initiation proneness. The priming phase was composed of reading some bogus statistics and a personal narrative. The bogus statistics described high probabilities of the occurrence of negative events. It was written in such a way that the statistics were from a survey of undergraduates, and the 
contents involved topics that undergraduates could easily engage in. The personal narrative was a short paragraph consisting of a stream of thoughts about possible negative future events. The narrative was written in an ambiguous way (in terms of context) to prevent the story from invoking any strong emotions. We used three sets of bogus statistics and personal narratives. One set concerned study/work, another concerned social relationships, and the third set concerned finances. There were three trials, each of which followed one of the three sets of bogus statistics and personal narratives. The order of presentation was randomized across participants.

For each trial of the worry initiation task, the ease of worry initiation was measured on a scale from 0 to $3(0=$ not initiated at either 1 st or 2 nd beep; $1=$ initiated worry only at 2 nd beep; $2=$ initiated worry only at 1 st beep, $3=$ initiated worry at both 1 st and 2 nd beeps). A worrisome thought that occurred while being instructed to pay attention to breathing is likely to reflect the ease of worry initiation rather than the difficulty of terminating worry. Individuals who reported initiating worry at both time points were considered to have greater ease of worry initiation than (a) individuals who reported initiating worry at only the first beep, who were considered to have greater ease of worry initiation than (b) individuals who took longer to initiate worrying and hence reported worry initiation at only the second beep, who were considered to have greater ease of worry than (c) individuals who never initiated worrying. The sum of the scores from three QT trials was used as the index of ease of worry initiation. The worry initiation task had good internal consistency $(\alpha=.71)$.

\section{Rumination Initiation Task}

The rumination initiation task was almost identical to the worry initiation task, with one exception -- the content of the bogus statistics and personal narrative during the priming phase differed. Specifically, the bogus statistics used for the worry initiation task described high 
probabilities of future negative events, whereas the bogus statistics used for the rumination initiation task described past negative events. The narrative used for the worry initiation task was mostly composed of a chain of thoughts concerning possible future negative events, whereas the narrative used for the rumination initiation task was mostly composed of a chain of thoughts concerning past negative events. The scoring scheme of the rumination initiation task was identical to that of the worry initiation task (see details in Appendix A).

\section{Worry Termination Task}

To measure worry termination, participants engaged in two trials of a thinking period, during which participants were instructed to worry about their worry topic (see details below). The procedure for the thinking period was adapted mainly from Eysenck's (1984) assessment of the worry process. Participants were first asked to think of three current worries. These worries were to be of moderate and comparable intensity. Participants wrote a brief description of each worry and categorized the content of their worry topics as work/study, social/relationships, physical, financial or other. They rated the intensity of each worry on a 100-point scale ranging from 0 (not worrying at all) to 100 (the most worrying thing you can possibly imagine). The experimenter reviewed the three worry topics and chose two that fell within a moderate range of intensity (between 40 and 80) and were distinct from each other. Any participants who mentioned more than two worries outside this range were asked to think of other moderateintensity worries.

The experimenter and the participant then discussed the first worry topic for around 1 minute to remind the participant of all salient aspects. This process was designed to make sure virtually all participants began worrying. During the thinking period, which lasted 3 minutes, participants were instructed to worry about the topic just discussed with their eyes closed. 
Participants were signaled six times (every 30 seconds) at which time they were to indicate what they were doing at the time of the signal. At each signal, participants were first asked, "Since the last beep, have you been worrying continuously? ${ }^{1,}$ Participants indicated one of the following: (1) Yes, I have been worrying continuously, (2) No, I stopped worrying, but then I started up worrying again, or (3) No, I stopped worrying and did not start again. They were then asked to indicate, since the last beep, how much of the time they had been worrying on a scale of 1 (not at all) to 5 (the entire time). At the end of the task, participants were asked how difficult they found it to stop worrying during the exercise on a scale of 1 (not at all) to 5 (extremely). This whole procedure, which was considered a trial, was repeated for the second worry topic.

For each trial, the frequency of continuing worrying was measured on a scale from 1 to 3 ( 1 = "No, I stopped worrying and did not start again"; 2 = "No, I stopped worrying, but then I started up worrying again"; 3 = "Yes, I have been worrying continuously"). The index of difficulty with worry termination was computed using the sum of the six items: three items per trial (i.e., the frequency of continuing worrying, the time spent on worrying, and difficulty stopping worrying) x 2 trials. The worry termination task had good internal consistency $(\alpha=.84)$.

\section{Rumination Termination Task}

The rumination termination task was almost identical to the worry termination task except for two things. First, instead of indicating worry topics, participants were asked to think of three current ruminations: things that happened in the past that currently concern them and that they have been dwelling on (e.g., something they did that they wish they had not done and/or something they did not do but wish they had). Two of the three rumination topics were selected for the rumination termination task (the ones that fell in the moderate range of intensity). During the thinking period, participants were instructed to dwell on the topic previously identified with 
their eyes closed. Second, the questions asked during the thinking period were structurally identical to those of the worry termination task except to probe difficulty with rumination termination. The same procedure, which was considered a trial, was repeated for the second rumination topic. The scoring scheme of the rumination termination task was identical to that of the worry termination task (see details in Appendix B).

\section{Ecological Momentary Assessment of Initiation and Termination of Worry/Rumination}

For EMA, we largely adopted Berenbaum et al.’s (2018) EMA method but expanded it to measure rumination in addition to worry. Survey signals were generated using Qualtrics software (2005, Provo) and were delivered as a text message through participants' smartphones. The text message contained a survey link that included a series of questions about worry and rumination. Participants were prompted six times per day over the course of five consecutive days. Participants were allowed to choose the time frame $(8 \mathrm{am}-11 \mathrm{pm}, 9 \mathrm{am}-12 \mathrm{am}, 10 \mathrm{am}-1 \mathrm{am}$ or $11 \mathrm{am}-2 \mathrm{am})$ they would prefer, all of which covered 15 hours per day. Prompts were randomly dispersed within two and a half hour time periods throughout the day, such that one message would occur within every two and a half hour time period (i.e., one message between 10AM 12:30PM, and the next message sent between 12:30PM - 3PM, etc). Prompts occurred as few as 150 and as many as 198 minutes apart $(\mathrm{M}=181, \mathrm{SD}=32)$. Participants were given up to 20 minutes to respond to each prompt and subsequent series of questions. When participants did not respond, the survey link for that prompt expired, recording missing data for that prompt. Participants responded to between $13(43.3 \%)$ and $30(100 \%)$ prompts $[\mathrm{M}=24.6(82 \%), \mathrm{SD}=$ 4.0] out of a total of 30 prompts. Response rate to prompts was not significantly correlated with either PSWQ $(r=.18, p>.2)$ or RRQ $(r=.22, p>.1)$ scores. 
At each prompt, participants were asked a set of worry and rumination related questions. The order of questions (worry vs. rumination) was counterbalanced. For worry, participants were first asked whether they had worried at any time since the last prompt. If they had worried, participants were asked: (a) during the most recent block of time (since the last message), whether they started worrying about anything new that they had not been worrying about during the preceding block of time $(0=n o, 1=y e s)$; $(\mathrm{b})$ during the most recent block of time (since the last message), whether they continued worrying about something that they had already been worrying about during the preceding block of time $(1=$ No, I have not been worrying about anything that I had already been worrying about during the preceding block of time; $2=Y e s, I$ have been worrying about something that I had already been worrying about during the preceding block of time, but it has been off and on; $3=$ Yes, I have been worrying about something that I had already been worrying about during the preceding block of time, and I have worried about it continuously); (c) since the last message, how difficult it has been to stop worrying $(0=$ not at all difficult to $5=I$ was unable to stop worrying $)$; and (d) since the last message, how much of the time had they been worrying?" $(0=$ never to $4=$ all of the time $)$. Due to the nature of the questions asked (using the time frame of "since the last message"), the first prompted message of the day did not include question (a) or question (b). The first question asked (i.e., whether they worried since they woke up) was instead used for assessing the initiation of worry. If participants reported not having worried since the last message, they were asked the same questions, but regarding the last time they had worried. These responses were not included in the analyses; they were included to discourage participants from reporting they had not worried as a strategy to have to answer fewer questions. 
The proportion of prompts on which participants had new worries (question a), which was adjusted based on the number of prompts to which they responded, was used as an index of ease of worry initiation. To measure continuing worries (question b), the responses were recoded as 0 or 1: both answer 1 ("No, I have not been worrying about anything that I had already been worrying about during the preceding block of time") and answer 2 ("Yes, I have been worrying about something that I had already been worrying about during the preceding block of time, but it has been off and on") were coded as 0 and answer 3 ("Yes, I have been worrying about something that I had already been worrying about during the preceding block of time, and I have worried about it continuously") was coded as 1 . Then, the summed score across prompts were divided by the number of responded prompts to calculate the proportion of prompts on which participants had continuing worries. Finally, as an index of difficulty with worry termination, a composite score was created by averaging the z-scores of the following variables that were highly correlated (correlations ranged from 0.6 to 0.8 ):1) proportion of prompts on which they had continuing worries (question b), adjusted based on the number of prompts responded to, 2) difficulty stopping worrying (question c), and 3) the time spent worrying (question d).

The rumination questions were structured in the exact same way as worry questions except to probe about participants' rumination pattern. Scoring scheme of the rumination questions was also identical to that of the worry questions (see details in Appendix C).

\section{RESULTS AND DISCUSSION}

The following variables were positively skewed (i.e., absolute value of the skewness statistic greater than two times the standard error of the skewness) and thus log-transformed: Lab rumination initiation, EMA worry initiation, EMA rumination initiation, EMA rumination termination. Descriptive statistics for all variables used can be found in Appendix D. 


\section{Differentiating worry vs. rumination}

As can be seen in Table $1^{1}$, the lab rumination measures of initiation and termination were both significantly correlated with the RRQ, $r=.37, p=.01$ and $r=.32, p=.03$, respectively, but not with the PSWQ, $r=-.02, p>.9$ and $r=.21, p>.1$ respectively. Similarly, the lab worry measures of initiation and termination were both significantly correlated with the PSWQ, $r=.30, p=.04$ and $r=.37, p=.01$, respectively, whereas the lab worry initiation was not significantly correlated with the RRQ, $r=.27, p>.07$ and only the lab worry termination was significantly correlated with the RRQ, $r=.32, p=.03$ (but to a lesser degree than with the PSWQ).

In comparison, the EMA measures were not differentially associated with the RRQ and PSWQ in the manner that was expected. Specifically, the EMA worry initiation was positively correlated with the RRQ, $r=.41, p=.005$, but not with the PSWQ, $r=.18, p>.2$. The EMA worry termination was not significantly correlated with either the PSWQ, $r=.27, p=.08$ or the RRQ, $r=.28, p=.064$. Although the EMA rumination termination was positively correlated with the RRQ, $r=.34, p=.034$, the EMA rumination initiation was not significantly correlated with either the PSWQ, $r=.08, p>.5$ or the RRQ, $r=.08, p>.6$.

In summary, overall, the lab measures were better differentiating worry from rumination than were the EMA measures.

\section{Differentiating the initiation vs. termination phases}

As can be seen in Table 2, the lab measures did not seem to differentiate the initiation vs. termination phases of worry and rumination very well. For example, lab worry initiation was strongly correlated with lab worry termination, $r=.59, p<.000$, and lab rumination initiation was positively correlated with lab rumination termination, $r=.33, p=.03$. 
On the other hand, consistent with Berenbaum et al. (2018), the EMA measures better differentiated the initiation vs. termination phases of worry and rumination. The strongest correlations were found between EMA worry initiation and rumination initiation, $r=.76, p$ $<.000$ and between EMA worry termination and rumination termination, $r=.67, p<.000$. EMA worry initiation was not significantly correlated with EMA worry termination, $r=.27, p=.08$, and EMA rumination initiation was not significantly correlated with rumination termination, $r$ $=.09, p=.6$.

In summary, the present findings suggest that, in general, the lab measures were reasonably able to differentiate worry from rumination, but not initiation from termination. These findings are consistent with the possibility that there are at least some meaningful differences between worry and rumination. Consequently, in Study 2, we collected data from an independent sample in which we obtained lab measures, attention bias measures, and questionnaires to further explore potential distinctions between worry and rumination. We also found that the EMA measures were able to distinguish between initiation and termination (consistent with Berenbaum et al., 2018), but not between worry and rumination.

\section{STUDY 2}

The goal of Study 2 was to examine the relationship between attentional bias and both worry and rumination, focusing on: (1) whether worry and rumination are associated with attentional biases regarding different types of content (physical threat/danger vs. loss/failure); (2) whether attention is drawn toward or away from specific types of stimuli; and (3) whether the initiation and termination phases of worry and rumination have distinctive or similar patterns of attentional bias.

\section{METHODS}




\section{Participants}

Participants were 223 undergraduate students $(69.8 \%$ female; mean age $=18.9$ years $)$. The majority of participants (59.0\%) were White, followed by $19.5 \%$ Asian, $11.8 \%$ African American, and 5.2\% biracial; 4.5\% chose to describe themselves as "other." All participants completed a voluntary informed consent at the start of the session and received monetary compensation for their participation. The research protocol was approved by the university institutional review board.

\section{Measures of Worry and Rumination}

The same laboratory tasks (worry initiation, worry termination, rumination initiation, and rumination termination) were used as in Study 1 . We additionally administered the PSWQ and the RRQ.

\section{Measures of Attentional Bias for Physical Threat/Danger and Loss/Failure}

In order to test our hypothesis that worry and rumination would be differentially associated with biased attention to different types of content (physical threat vs. loss/failure), we implemented a dot-probe task using both types of content as stimuli. Since there was no previous study differentiating the content of the stimuli in such a way (i.e., physical threat vs. loss/failure) within the same paradigm, we used the following procedure. A total of 253 pictures were selected from the International Affective Picture System (IAPS; Lang, Bradley, \& Cuthbert, 1997) and from a sorted source on the web. We selected pictures that potentially fall in one of the following stimulus types: physical threat/danger (e.g., man with knife), loss/failure (e.g., people crying), and neutral (e.g., woman reading). These pictures were rated independently by seven raters using the Valence and Arousal scales of the Self-Assessment Manikin (SAM; Bradley \& Lang, 1994) which ranged from 1 (low pleasure, low arousal) to 9 (high pleasure, 
high arousal). Each picture was rated on two additional scales, physical threat/danger and loss/failure, on a scale of 1 (not at all relevant) to 5 (extremely relevant). Pictures were categorized as physical threat/danger if they received an average rating of greater than 3.5 on the physical threat/danger scale, less than 2.5 on the loss/failure scale, and less than 3.5 on the valence scale. Pictures were categorized as loss/failure if they received an average rating of greater than 3.5 on the loss/failure scale, less than 2.5 on the physical threat/danger scale, and less than 3.5 on the valence scale. Finally, pictures were categorized as neutral if they received an average rating of less than 2.5 on both physical threat/danger and loss/failure scales, and higher than 4.5 on the valence scale. These selection criteria resulted in the selection of 120 pictures (48 from the IAPS; 72 from the web); 24 pictures for physical threat/danger, 24 pictures for loss/failure, and 72 pictures for neutral. The descriptive statistics of the physical thereat/danger, loss/failure, valence, and arousal ratings are reported in Appendix E. Another 24 neutral pictures were selected for the practice trials. In line with the procedure of Mogg et al. (2000), three types of stimulus pairs were created: physical threat/danger - neutral (24 pairs), loss/failure - neutral (24 pairs), neutral - neutral (24 pairs). The pictures were carefully matched with respect to valence and arousal ratings and the presence of a person and concreteness. Pictures had a height of $4.6 \mathrm{~cm}$ and a mean width of $6.5 \mathrm{~cm}$. The dot-probe task was programmed and presented using E-Prime (Schneider, Eschman, \& Zuccolotto, 2002).

Each of the 48 emotion picture pairs (physical threat/danger, loss/failure) was presented four times and each of the 24 neutral picture pairs was presented two times, for a total of 240 trials, which were presented in a new, fully randomized order for each participant. Each trial started with a fixation cross in the middle of the screen for $500 \mathrm{~ms}$. Then, two pictures appeared, one $2.2 \mathrm{~cm}$ above and one $2.2 \mathrm{~cm}$ below, the center of the screen for $500 \mathrm{~ms}$. Following the 
offset of the pictures, a blank screen showed for $14 \mathrm{~ms}$ and a single dot or a double dot appeared in the center of the screen location where one of the pictures had been and remained on the screen until the participant pressed one of the two keys on the keyboard to indicate whether the target is a single dot or a double dot. The accuracy and latency of each response was recorded. The inter-trial interval was one of the following, in a random order: 500, 750, 1000, and $1250 \mathrm{~ms}$. The emotional stimulus pictures (physical threat/danger, loss/failure) appeared in the upper and the lower positions with equal probability, with the matched neutral picture of each pair appearing in the other position. The target (a dot or a double dot) was also presented in both positions with equal probability.

Participants were told that the target could appear in the upper or lower position on the screen and were instructed to indicate whether the target was a single dot or a double dot as quickly and accurately as possible by pressing the left arrow key if the target was a single dot

and by pressing the right arrow key if the target was a double dot. Participants first completed 12 practice trials before completing the actual task.

\section{Procedures}

All participants completed all of the following tasks: four laboratory tasks measuring the initiation and termination of worry and rumination, dot-probe task, and questionnaires. To minimize fatigue and potential transfer effects between tasks, the study was conducted in two separate sessions, each held on a different day. The order of tasks was counterbalanced.

\section{RESULTS AND DISCUSSION}

\section{Data Processing}

Dot-probe task. Data from 3 participants were removed from analysis because their overall accuracy was less than $80 \%$. After exclusion, overall accuracy was $96.5(\mathrm{SD}=0.03) \%$. We 
excluded from analysis trials in which the RTs were shorter than $200 \mathrm{~ms}$, exceeded 2,500 ms, were 3SDs above each participant's mean, or were incorrect.

Attentional bias indices were calculated for each stimulus type (i.e., physical threat/danger and loss/failure) using MacLeod and Mathews' (1988) method where RTs on congruent trials are subtracted from RTs on incongruent trials. Thus, an index for attentional bias to physical threat/danger was created by subtracting RTs on congruent trials from RTs on incongruent trials for physical/danger stimuli (i.e., Threat/danger Incongruent RTs Threat/danger Congruent RTs). A positive attentional bias score indicates attention bias toward the threatening pictures, a negative attentional bias score indicates attention bias away from threat, and zero indicates no attentional bias. Similarly, an index for attentional bias to loss/failure was created by subtracting RTs on congruent trials from RTs on incongruent trials for loss/failure stimuli (i.e., Loss/Failure Incongruent RTs - Loss/Failure Congruent RTs). Descriptive statistics of attentional bias indices are reported in Appendix F.

Worry and Rumination Measures. Lab worry initiation and lab rumination initiation measures were positively skewed (i.e., absolute value of the skewness statistic greater than two times the standard error of the skewness) and thus log-transformed. Descriptive statistics for all the laboratory variables and questionnaires are reported in Appendix G.

\section{Correlations between Attentional Bias Indices and Laboratory and Questionnaire Measures of Worry and Rumination}

As shown in Table 3, worry initiation was associated with attention bias away from threat, $r=-.14, p=.04$, such that the more avoidance of threat stimuli, the greater the likelihood of initiating worry. In addition, though not significant, there was a trend for worry termination to 
also be associated with bias away from threat, $r=-.12, p=.08$. Neither worry initiation nor worry termination was correlated with attentional bias to loss/failure, $p \mathrm{~s}>.4$.

Rumination termination was associated with attention bias toward loss/failure, $r=.16, p$ $=.019$, such that the more bias towards loss/failure stimuli, the greater the difficulty terminating rumination. Rumination initiation was not correlated with attentional bias to loss/failure, $r=.05$, $p>$.4. Neither rumination initiation nor rumination termination was correlated with attentional bias to threat, $p \mathrm{~s}>.1$.

In addition, analyses using the data analytic approach ${ }^{2}$ recommended by Meng et al. (1992) indicate that worry and rumination show differential content-specificity in their attentional bias. That is, worry initiation was significantly more strongly correlated with a bias index for threat than with a bias index for loss/failure, $\mathrm{z}=-2.0, p=.046$. Rumination termination was significantly more strongly correlated with a bias index for loss/failure than with a bias index for threat, $\mathrm{z}=-2.81, p=.005$. Neither worry termination nor rumination initiation showed differences in the magnitude of correlations with a bias index for threat vs. a bias index for loss/failure, $p \mathrm{~s}>.1$.

Lastly, neither the PSWQ nor the RRQ were significantly correlated with any of the attentional bias measures, $p \mathrm{~s}>.3$.

In summary, building upon the distinction between worry vs. rumination observed using the lab measures in Study 1, the findings from Study 2 suggest different patterns of attentional bias associated with worry and rumination.

\section{GENERAL DISCUSSION}

Taken together, the results of the two studies suggest that there are some meaningful differences between worry and rumination. In Study 1, we found that the laboratory measures 
were reasonably able to differentiate worry from rumination. In addition, findings from Study 2 suggest that worry and rumination can be differentiated in terms of their attention to different contents (threat vs. loss/failure). That is, worry was associated with bias away from threat, whereas rumination was associated with bias toward loss/failure. It is notable that not only were worry and rumination associated with attentional biases to different content, the patterns of the associations (toward or away from stimuli) were in opposite directions.

Although previous studies with anxiety disorders and MDD have shown contentspecificity of attentional biases (i.e., attentional bias to threat in anxiety disorders and attentional bias to dysphoric information in MDD) (Hankin, Gibb, Abela, \& Flory, 2010; Mathews \& MacLeod, 2005), the present study is the first to demonstrate that worry and rumination show differential content-specificity in their attentional bias. Furthermore, this is the first study demonstrating that rumination is more broadly associated with a bias towards information related to a theme of loss/failure rather than dysphoric information (e.g., sad faces) only. These findings are consistent with the existing conceptualization that describes worry as repeated ideation focused on to-be-avoided threats, and rumination as focused on to-be-understood loss or failure (Mathews \& MacLeod, 2005; Smith \& Alloy, 2009). In addition, the current findings are particularly relevant to the Research Domain Criteria (RDoC) initiative, which highlights threat and loss as key constructs, each of which are characterized by biased attention processing in anxiety and depression (Dillon et al., 2014; Gibb, McGeary, \& Beevers, 2016; Mennin \& Fresco, 2013). Whereas previous research focused on alterations in threat and reward processing in anxiety and depression, the current findings suggest that worry and rumination are also differentially associated with threat vs. reward processing, similar to anxiety and depression. Accumulating evidence suggests that attention training may benefit individuals with emotional 
disorders (Cisler \& Koster, 2010). The results of the present study raise the possibility that the success of attention training may be enhanced by attending to the contents of the stimuli used (e.g., utilizing different contents of stimuli depending on individuals' primary pattern of negative repetitive thinking).

It is important to note that the direction of relationship between worry and attention bias found in the current study (i.e., worry initiation is associated with threat avoidance) is in constrast with prior findings that demonstrated that high worriers and individuals with GAD show an increased attention bias towards threat (Goodwin, Yiend, et al., 2017; Mathews \& MacLeod, 2005). It is possible that the differences in sample characteristics (i.e., non-clinical, unselected individuals vs. pathological, high worriers) may have contributed to this discrepancy. That is, individuals who worry more easily show greater avoidance of threat, but as their worry symptoms worsen and reach the level of clinical significance, the nature of attention bias changes such that their attention is oriented to threat. Supporting this account, studies have shown that high worriers and non-worrying high trait anxious individuals (e.g., Engel et al., 2005; Paulesu et al., 2010) show distinct neural characteristics. Nonetheless, future longitudinal work is warranted to examine the potential changes in attention bias patterns as a function of clinical severity of worry symptoms. In addition, we cannot rule out the possibility that other features of the study (e.g., types of stimuli used, duration of the stimuli) may have contributed to this discrepancy. A few studies have found that, in a probe task using neutral and threatening stimuli, higher stimulus threat levels led to faster detection (vigilance), whereas low levels of stimulus threat (the types of stimuli we used) led to slowed detection of probes in the same location (avoidance) (Koster, Crombez, Verschuere, \& De Houwer, 2006; Wilson \& MacLeod, 2003). ${ }^{3}$ In addition, given that the attention bias measures using dot-probe tasks provide only a snapshot 
of attention at one point in time (Bradley, Mogg, Falla, \& Hamilton, 1998; Kellough, Beevers, Ellis, \& Wells, 2008), it will be important to examine the changing course of attention over time. This could be achieved using different stimulus durations or different methods, such as eyetracking.

The avoidance theory of worry (Borkovec, 1994) suggests that worry is an avoidance response that prevents individuals from adequately processing situationally relevant information and deploying adaptive coping responses, which leads to pathological distress and worsening of the symptoms. In contrast, rumination theorists suggest that focusing one's attention on stressors and symptoms is more maladaptive than distracting oneself from thinking about them (NolenHoeksema, 1998). Based on these theories, the current findings suggest that both worry and rumination are associated with attention patterns (i.e., avoiding threat, focusing on loss/failure) that have been described as maladaptive. It is possible that the attentional bias patterns associated with worry and rumination may play a role in the development of emotional disturbances, such as anxiety and depression. They are also consistent with the view that worry and rumination differ with regard to at least some attentional processes (Beck, 2008; Clark et al., 1989).

We hypothesize that both worry laboratory tasks largely tap individual differences in the degree to which potential sources of threat/danger are considered significant - in other words, they are largely indices of threat perception. Along the same lines, we hypothesize that both rumination laboratory tasks largely tap individual differences in the degree to which potential sources of loss/failure are considered significant. Such an account can explain several findings across the two studies. First, it can explain why the laboratory tasks distinguished between worry and rumination. The main difference between the worry and rumination lab measures was eliciting negative thoughts using different types of content (threat/danger vs. loss/failure). 
Specifically, we used different types of prompt content for the initiation tasks, and different types of topic content for the termination tasks. Second, this account can also explain why the laboratory tasks did not distinguish between initiation and termination. The recent modification of the IT model of worrying (Berenbaum et al., 2018) proposes that threat perception influences both the initiation and termination of worrying. This shared characteristic may have been reflected on both the initiation and the termination lab tasks. Third, this account explains why the two worry tasks did not differ significantly in their associations with attentional bias to threat/danger and why the two rumination tasks did not differ significantly in their associations with attentional bias to loss/failure. Because both worry initiation and termination tasks captured individual differences in perception of threat, they may have shown similar patterns of association with biased attention to threat. Similarly, because both rumination initiation and termination tasks captured individual differences in perception of loss/failure, they may have shown similar patterns of association with biased attention to loss/failure. Finally, given that we would expect attentional bias to be associated most specifically with perceptions of threat/danger and loss/failure (as opposed to other aspects of worrying and ruminating), this account of the lab tasks can explain why the lab tasks were associated with attentional bias whereas questionnaire measures of worry and rumination were not. It is possible that questionnaires are not as good as lab tasks in capturing individual differences in the degree to which potential sources of threat or loss/failure are considered significant. It is worth noting that prior findings investigating the link between attention bias and questionnaire measures of worry and rumination are largely mixed (Engels et al., 2007; Oathes et al., 2011; Sass et al., 2010).

We acknowledge some limitations of the present research. There is undoubtedly room for improving the present lab tasks as well as the EMA measures. In addition to considering 
modifications to the instructions, it will be worth adding or modifying some of the questions. For example, for the worry termination task, one could potentially use additional questions to reduce any ambiguity (e.g., Are you worrying? Did you try to stop worrying? If so, how difficult was it to stop worrying?). In addition, for EMA, one can potentially collect information about what participants were worrying about to understand the nature and pattern of their worries. Because we used a sample of college students, it likely included few, if any, individuals with clinically debilitating levels of worrying/ruminating. Thus, it will be important for future studies to examine whether the current findings are replicated, or if different patterns emerge in samples with greater representation of the extreme end of the worrying and ruminating continua. In addition, it is important to note that, in the dot-probe administered in Study 2, we used stimuli specifically related to physical threat or danger (but not social or other types of threat) in order to differentiate them from the loss/failure stimuli. Because of the inherent difference in threat vs. loss/failure stimuli, arousal levels of stimuli are not equated, and this should be noted as a limitation. Future research using different sets of stimuli that capture a broader range of worry and rumination topics is warranted. In addition, although a dot-probe is one of the most frequently used attentional bias measures, it has been criticized due to its poor psychometric properties (Schmukle, 2005). Thus, future studies may supplement traditional measures of attentional bias (e.g., dot-probe) with additional measures of cognitive processing and performance, including implicit measures like eye tracking and psychophysiological measures such as EEG and neuroimaging. Finally, the present findings are based on a cross-sectional design. Future research is warranted to investigate the potential causal relationships of distinctive attentional processes and worry and rumination using rigorous longitudinal designs and experimental manipulations. Despite its limitations, the current study adds to the evidence that 
there are some meaningful differences between worry and rumination, and provides clues to better understanding them, their roles in emotional disorders, and how they may be best targeted in treatment. 
Attentional Biases, Worry and Rumination

\section{REFERENCES}

Bar-Haim, Y., Lamy, D., Pergamin, L., Bakermans-Kranenburg, M. J., \& Van Ijzendoorn, M. H. (2007). Threat-related attentional bias in anxious and nonanxious individuals: a metaanalytic study. Psychological bulletin, 133(1), 1.

Beck, A. T. (2008). The evolution of the cognitive model of depression and its neurobiological correlates. American Journal of Psychiatry, 165(8), 969-977.

Beck, A. T., Brown, G., Steer, R. A., Eidelson, J. I., \& Riskind, J. H. (1987). Differentiating anxiety and depression: A test of the cognitive content-specificity hypothesis. Journal of Abnormal Psychology, 96(3), 179.

Beck, A. T., \& Haigh, E. A. (2014). Advances in cognitive theory and therapy: the generic cognitive model. Annu Rev Clin Psychol, 10, 1-24.

Berenbaum, H. (2010). An initiation-termination two-phase model of worrying. Clin Psychol Rev, 30(8), 962-975. doi:10.1016/j.cpr.2010.06.011

Berenbaum, H., Chow, P. I., Flores Jr, L. E., Schoenleber, M., Thompson, R. J., \& Most, S. B. (2018). A test of the initiation-termination model of worry. Journal of Experimental Psychopathology, 9(1), 2043808718778965.

Borkovec, T. D. (1994). The nature, functions, and origins of worry. In G. Davey \& F. Tallis (Eds.), Worrying: Perspectives on theory, assessment and treatment (pp. 5-33). England: Wiley; Oxford.

Borkovec, T. D., Robinson, E., Pruzinsky, T., \& DePree, J. A. (1983). Preliminary exploration of worry: Some characteristics and processes. Behaviour Research and Therapy, 21(1), 9-16. 
Bradley, B. P., Mogg, K., Falla, S. J., \& Hamilton, L. R. (1998). Attentional bias for threatening facial expressions in anxiety: Manipulation of stimulus duration. Cognition \& Emotion, 12(6), 737-753.

Bradley, M. M., \& Lang, P. J. (1994). Measuring emotion: the self-assessment manikin and the semantic differential. J Behav Ther Exp Psychiatry, 25(1), 49-59.

Cisler, J. M., \& Koster, E. H. (2010). Mechanisms of attentional biases towards threat in anxiety disorders: An integrative review. Clin Psychol Rev, 30(2), 203-216. doi:10.1016/j.cpr.2009.11.003

Clark, D. A., Beck, A. T., \& Brown, G. (1989). Cognitive mediation in general psychiatric outpatients: A test of the content-specificity hypothesis. Journal of Personality and Social Psychology, 56(6), 958.

Dillon, D. G., Rosso, I. M., Pechtel, P., Killgore, W. D., Rauch, S. L., \& Pizzagalli, D. A. (2014). Peril and pleasure: An RDOC-inspired examination of threat responses and reward processing in anxiety and depression. Depression and Anxiety, 31(3), 233-249.

Donaldson, C., Lam, D., \& Mathews, A. (2007). Rumination and attention in major depression. Behav Res Ther, 45(11), 2664-2678. doi:10.1016/j.brat.2007.07.002

Engel, S. G., Wonderlich, S. A., Crosby, R. D., Wright, T. L., Mitchell, J. E., Crow, S. J., \& Venegoni, E. E. (2005). A study of patients with anorexia nervosa using ecologic momentary assessment. Int J Eat Disord, 38(4), 335-339. doi:10.1002/eat.20184

Engels, A. S., Heller, W., Mohanty, A., Herrington, J. D., Banich, M. T., Webb, A. G., \& Miller, G. A. (2007). Specificity of regional brain activity in anxiety types during emotion processing. Psychophysiology, 44(3), 352-363. 
Eysenck, M. W. (1984). Anxiety and the worry process. Bulletin of the Psychonomic Society, 22(6), 545-548.

Gibb, B. E., McGeary, J. E., \& Beevers, C. G. (2016). Attentional biases to emotional stimuli: Key components of the RDoC constructs of sustained threat and loss. American Journal of Medical Genetics Part B: Neuropsychiatric Genetics, 171(1), 65-80.

Goodwin, H., Eagleson, C., Mathews, A., Yiend, J., \& Hirsch, C. (2017). Automaticity of Attentional Bias to Threat in High and Low Worriers. Cognit Ther Res, 41(3), 479-488.

Goodwin, H., Yiend, J., \& Hirsch, C. R. (2017). Generalized Anxiety Disorder, worry and attention to threat: A systematic review. Clin Psychol Rev, 54, 107-122.

Grafton, B., Southworth, F., Watkins, E., \& MacLeod, C. (2016). Stuck in a sad place: Biased attentional disengagement in rumination. Emotion, 16(1), 63.

Hankin, B. L., Gibb, B. E., Abela, J. R., \& Flory, K. (2010). Selective attention to affective stimuli and clinical depression among youths: role of anxiety and specificity of emotion. Journal of Abnormal Psychology, 119(3), 491.

Joormann, J., Dkane, M., \& Gotlib, I. H. (2006). Adaptive and maladaptive components of rumination? Diagnostic specificity and relation to depressive biases. Behav Ther, 37(3), 269-280. doi:10.1016/j.beth.2006.01.002

Kellough, J. L., Beevers, C. G., Ellis, A. J., \& Wells, T. T. (2008). Time course of selective attention in clinically depressed young adults: An eye tracking study. Behaviour Research and Therapy, 46(11), 1238-1243.

Kendall, P. C., \& Ingram, R. E. (1989). Cognitive-behavioral perspectives: Theory and research on depression and anxiety. 
Kirkegaard Thomsen, D. (2006). The association between rumination and negative affect: A review. Cognition and Emotion, 20(8), 1216-1235.

Koster, E. H., Crombez, G., Verschuere, B., \& De Houwer, J. (2006). Attention to threat in anxiety-prone individuals: Mechanisms underlying attentional bias. Cognit Ther Res, $30(5), 635-643$.

Koster, E. H., De Raedt, R., Goeleven, E., Franck, E., \& Crombez, G. (2005). Mood-congruent attentional bias in dysphoria: maintained attention to and impaired disengagement from negative information. Emotion, 5(4), 446-455. doi:10.1037/1528-3542.5.4.446

Lang, P. J., Bradley, M. M., \& Cuthbert, B. N. (1997). International affective picture system (IAPS): Technical manual and affective ratings. NIMH Center for the Study of Emotion and Attention, 39-58.

Martin, L. L., Tesser, A., \& McIntosh, W. D. (1993). Wanting but not having: The effects of unattained goals on thoughts and feelings.

Mathews, A., \& MacLeod, C. (2005). Cognitive vulnerability to emotional disorders. Annu Rev Clin Psychol, 1, 167-195. doi:10.1146/annurev.clinpsy.1.102803.143916

McEvoy, P. M., Watson, H., Watkins, E. R., \& Nathan, P. (2013). The relationship between worry, rumination, and comorbidity: evidence for repetitive negative thinking as a transdiagnostic construct. J Affect Disord, 151(1), 313-320.

doi:10.1016/j.jad.2013.06.014

Mennin, D. S., \& Fresco, D. M. (2013). What, me worry and ruminate about DSM-5 and RDoC? The importance of targeting negative self-referential processing. Clinical Psychology: Science and Practice, 20(3), 258-267. 
Meyer, T. J., Miller, M. L., Metzger, R. L., \& Borkovec, T. D. (1990). Development and validation of the penn state worry questionnaire. Behaviour Research and Therapy, 28(6), 487-495.

Mogg, K., Bradley, B. P., Williams, R., \& Mathews, A. (1993). Subliminal processing of emotional information in anxiety and depression. Journal of Abnormal Psychology, 102(2), 304.

Mor, N., \& Winquist, J. (2002). Self-focused attention and negative affect: a meta-analysis. Psychological bulletin, 128(4), 638.

Nolen-Hoeksema, S. (1998). The other end of the continuum: The costs of rumination. Psychological Inquiry, 9(3), 216-219.

Nolen-Hoeksema, S., Wisco, B. E., \& Lyubomirsky, S. (2008). Rethinking rumination. Perspectives on psychological science, 3(5), 400-424.

Oathes, D. J., Siegle, G. J., \& Ray, W. J. (2011). Chronic worry and the temporal dynamics of emotional processing. Emotion, 11(1), 101.

Paulesu, E., Sambugaro, E., Torti, T., Danelli, L., Ferri, F., Scialfa, G., . . Sassaroli, S. (2010). Neural correlates of worry in generalized anxiety disorder and in normal controls: a functional MRI study. Psychol Med, 40(1), 117-124.

Rapee, R. M. (1993). The utilisation of working memory by worry. Behaviour Research and Therapy, 31(6), 617-620.

Ruscio, A. M., \& Borkovec, T. D. (2004). Experience and appraisal of worry among high worriers with and without generalized anxiety disorder. Behav Res Ther, 42(12), 14691482. doi:10.1016/j.brat.2003.10.007 
Ruscio, A. M., Seitchik, A. E., Gentes, E. L., Jones, J. D., \& Hallion, L. S. (2011). Perseverative thought: a robust predictor of response to emotional challenge in generalized anxiety disorder and major depressive disorder. Behav Res Ther, 49(12), 867-874. doi:10.1016/j.brat.2011.10.001

Sass, S. M., Heller, W., Stewart, J. L., Silton, R. L., Edgar, J. C., Fisher, J. E., \& Miller, G. A. (2010). Time course of attentional bias in anxiety: emotion and gender specificity. Psychophysiology, 47(2), 247-259.

Schmukle, S. C. (2005). Unreliability of the dot probe task. European Journal of Personality, 19(7), 595-605.

Schneider, W., Eschman, A., \& Zuccolotto, A. (2002). E-Prime: User's guide: Psychology Software Incorporated.

Segerstrom, S. C., Stanton, A. L., Alden, L. E., \& Shortridge, B. E. (2003). A multidimensional structure for repetitive thought: what's on your mind, and how, and how much? J Pers Soc Psychol, 85(5), 909-921. doi:10.1037/0022-3514.85.5.909

Sheehan, D., Janavus, J., Baker, R., Harnett-Sheehan, K., Knapp, E., \& Sheehan, M. (2001). MINI Plus-Mini International Neuropsychiatric Interview. Brazilian version 5.0. 0. DSM $I V$.

Shiffman, S., Stone, A. A., \& Hufford, M. R. (2008). Ecological Momentary Assessment. Annu Rev Clin Psychol, 4(1), 1-32. doi:10.1146/annurev.clinpsy.3.022806.091415

Smith, J. M., \& Alloy, L. B. (2009). A roadmap to rumination: a review of the definition, assessment, and conceptualization of this multifaceted construct. Clin Psychol Rev, 29(2), 116-128. doi:10.1016/j.cpr.2008.10.003 
Southworth, F., Grafton, B., MacLeod, C., \& Watkins, E. (2017). Heightened ruminative disposition is associated with impaired attentional disengagement from negative relative to positive information: support for the "impaired disengagement" hypothesis. Cognition and Emotion, 31(3), 422-434.

Stokes, C., \& Hirsch, C. R. (2010). Engaging in imagery versus verbal processing of worry: Impact on negative intrusions in high worriers. Behaviour Research and Therapy, 48(5), 418-423.

Trapnell, P. D., \& Campbell, J. D. (1999). Private self-consciousness and the five-factor model of personality: distinguishing rumination from reflection. Journal of Personality and Social Psychology, 76(2), 284.

Wanke, M., \& Schmid, J. (1996). Rumination: When AII EIse FaiIs. Ruminative thoughts, 9, 177.

Watkins, E. R. (2008). Constructive and unconstructive repetitive thought. Psychol Bull, 134(2), 163-206. doi:10.1037/0033-2909.134.2.163

Wilson, E., \& MacLeod, C. (2003). Contrasting two accounts of anxiety-linked attentional bias: selective attention to varying levels of stimulus threat intensity. Journal of Abnormal Psychology, 112(2), 212. 


\section{Footnotes}

${ }^{1}$ Using Meng's (1992) analytic approach, only one of the eight pairs of correlations -i.e., the correlations of lab rumination initiation with PSWQ vs. RRQ - was significantly different, $z=-$ $2.19, p=.03$, whereas the other pairs of correlations were not significantly different, $p s>.1$. The absence of statistical significance is likely a reflection of the small sample size $(n=46)$.

${ }^{2}$ Extending Dunn and Clark's (1969) work, Meng's et al. (1992) data analytic approach utilizes the Fisher $\mathrm{z}$ transformation to test for the significance of the difference between two correlation coefficients.

${ }^{3}$ For the dot-probe task, we did not use the types of threat stimuli Koster et al. (2006) defined as highly threatening (e.g., mutilated face), but rather used threat stimuli that corresponded to what they defined as mildly threatening (e.g., man with knife). 
Table 1. Correlations Between Laboratory/EMA Measures and Questionnaires of Worry and Rumination

\begin{tabular}{lcc}
\hline & PSWQ & RRQ \\
\hline Lab Measures & & \\
Worry & $.30^{*}$ & .27 \\
$\quad$ Initiation & $.37^{*}$ & $.32^{*}$ \\
$\quad$ Termination & -.02 & $.37^{*}$ \\
Rumination & .21 & $.32^{*}$ \\
$\quad$ Initiation & & \\
Termination & & $.41^{* *}$ \\
EMA Measures & .18 & .28 \\
Worry & .27 & .08 \\
Initiation & & $.34^{*}$ \\
Termination & .08 & \\
Rumination & .10 & \\
Initiation & Termination &
\end{tabular}

Note: $* p<0.05, * * p<0.01$, two-tail 
Table 2. Correlations Between Laboratory and EMA Measures of Worry and Rumination

\begin{tabular}{|c|c|c|c|c|c|c|c|c|}
\hline & \multicolumn{4}{|c|}{ Lab Measures } & \multicolumn{4}{|c|}{ EMA Measures } \\
\hline & \multicolumn{2}{|c|}{ Worry } & \multicolumn{2}{|c|}{ Rumination } & \multicolumn{2}{|c|}{ Worry } & \multicolumn{2}{|c|}{ Rumination } \\
\hline & Initiation & Termination & Initiation & Termination & Initiation & Termination & Initiation & Termination \\
\hline \multicolumn{9}{|l|}{ Lab Measures } \\
\hline \multicolumn{9}{|l|}{ Worry } \\
\hline Initiation & --- & & & & & & & \\
\hline Termination & $.59^{* * *}$ & --- & & & & & & \\
\hline \multicolumn{9}{|l|}{ Rumination } \\
\hline Initiation & $.39^{* *}$ & .24 & -- & & & & & \\
\hline Termination & .28 & .20 & $.33^{*}$ & --- & & & & \\
\hline \multicolumn{9}{|l|}{ EMA Measures } \\
\hline \multicolumn{9}{|l|}{ Worry } \\
\hline Initiation & .10 & $.32^{*}$ & .15 & .07 & -- & & & \\
\hline Termination & $.30^{*}$ & $.33^{*}$ & $.30 *$ & $.50^{* *}$ & .27 & -— & & \\
\hline \multicolumn{9}{|l|}{ Rumination } \\
\hline Initiation & .11 & .27 & .19 & .07 & $.76^{* * *}$ & .18 & - & \\
\hline Termination & .02 & .15 & .20 & $.42^{* *}$ & .28 & $.67^{* * *}$ & .09 & - \\
\hline
\end{tabular}


Table 3. Correlations between Attentional Bias Indices and Laboratory and Questionnaire Measures of Worry and Rumination

\begin{tabular}{|c|c|c|c|c|}
\hline & \multicolumn{2}{|c|}{$\begin{array}{c}\text { Bias Index for Physical } \\
\text { Threat/Danger }\end{array}$} & \multicolumn{2}{|c|}{ Bias Index for Loss/Failure } \\
\hline & Pearson's $r$ & $p$-value & Pearson's $r$ & $p$-value \\
\hline \multicolumn{5}{|l|}{ Lab Measures } \\
\hline Worry Initiation & $-.14 *$ & .048 & .05 & .492 \\
\hline Worry Termination & -.12 & .080 & .01 & .892 \\
\hline Rumination Initiation & -.04 & .567 & .05 & .488 \\
\hline Rumination Termination & -.10 & .153 & $.16^{*}$ & .019 \\
\hline \multicolumn{5}{|l|}{ Questionnaires } \\
\hline Worry (PSWQ) & -.02 & .738 & -.06 & .384 \\
\hline Rumination (RRQ & -.03 & .652 & .06 & .367 \\
\hline
\end{tabular}

Note: ${ }^{*} p<0.05$, two-tail 